sei „nicht zu ermitteln“ (S. 217). Ähnlich verwirrend ist, dass die Gesprächspartner in den abgedruckten Interviews darauf hinweisen, dass die Bedeutung von Referentenposten für den parteiinternen Aufstieg überbewertet sei (S. 92 f., S. 199 f., S. 289), Leif in seinem Fazit dann aber anhand von zwei Beispielen aus dem Bund und dem Land Hessen zu dem Ergebnis kommt, hier gelte das Prinzip „Aufstieg garantiert“ (S. 434).

Die Lösungsansätze und Empfehlungen für die Zukunft bieten wenig Neues und werden kaum reflektiert. Leif nennt die Einführung „direkter Demokratie-Partizipation “ durch Plebiszite oder Volksabstimmungen (S. 364 f.), das Aufstellen von Bürgerhaushalten (S. 368), den Einsatz von Vorwahlen zur Kandidatenaufstellung (S. 370) und natürlich die verstärkte Nutzung des Internets (S. 377). Sicherlich sind diese Instrumente geeignet, neue Möglichkeiten auch für Bürger zu schaffen, die sich spontan beteiligen möchten. Doch die genannten Reform-Instrumente würden den Niedergang der Parteien beschleunigen. Auf die Frage, wie diese in ihrer Funktion für das politische System erhalten werden können, haben derzeit weder die Parteien selbst noch die Politikwissenschaft überzeugende konkrete Antworten.

Das Buch ist keine wissenschaftliche Studie, denn weder genügt es in Aufbau und Methodik den entsprechenden Anforderungen, noch hält sich der Verfasser in angemessener Distanz mit eigenen Wertungen zurück. Er gleitet teilweise in pure Polemik ab, wenn er Politiker als ehrgeizige „Egomanen“ bezeichnet (S. 146), Berater in einem missratenen Wortspiel ohne weitere Erläuterung „(Ex)-Politiker, (Ex)-Banker, (Ex)-Perten“ (S. 343) nennt oder zum Abschluss des Buches plötzlich von „Cliquen und Claqueuren“ (S. 435) spricht. Es kommen überdies sehr viele anonyme Quellen („Insider“) zu Wort, Nachweise werden unvollständig geführt (vgl. S. 475), und ausgiebig wird aus nicht-öffentlichen Papieren zitiert - die Nachprüfbarkeit vieler Aussagen ist daher kaum gegeben. Auch ist das Buch nicht nur durchgängig journalistisch flott, sondern wohl auch etwas zu schnell geschrieben worden: Da enden Sätze im Nichts oder sind Umbrüche falsch gesetzt (S. 102, 140, 149), fehlen Buchstaben und ganze Wörter (S. 117, S. 122, S. 142, S. 188) und sind Fachbegriffe schlicht falsch geschrieben (etwa „Refraiming“, S. 148, oder „Dopik“, S. 179); sogar der im Literaturverzeichnis hervorgehobene Verweis auf eine ausführliche InternetBibliographie zum Thema ist grob fehlerhaft. Dennoch kann der Band eine Reihe von Anregungen liefern, und insbesondere die vielfach und ausführlich zitierten internen Dokumente bieten für Forschung und Lehre Einblicke und Ansatzpunkte zum weiteren Nachhaken und Diskutieren.

Sven T. Siefken

\title{
Jahrbuch Extremismus und Demokratie: lohnende Lektüre
}

Backes, Uwe und Eckhard Jesse (Hrsg.): Jahrbuch Extremismus \& Demokratie 2007, 19. Jahrgang, Nomos Verlagsgesellschaft, Baden-Baden 2008, 532 Seiten, € 49,-.

Mit der einleitenden Bemerkung: „Das neue Jahrtausend hat eine merkliche Verstärkung der zentrifugalen Kräfte des deutschen Parteiensystems gebrach"“, verweisen die Herausgeber des Jahrbuchs Extremismus und Demokratie unmittelbar auf die politische Relevanz der von ihnen nunmehr bereits seit zwei Jahrzehnten zusammengestellten Analysen zu den 
Rändern des politischen Spektrums. Der 2008 erschienene, 532 Seiten starke Band gliedert sich wie seine Vorgänger in Dokumentationen und Analysen einerseits (275 Seiten), Darstellungen zu und Auseinandersetzungen mit einschlägiger Literatur andererseits (212 Seiten) sowie ergänzende Verzeichnisse und Hinweise. Autoren sind etablierte und jüngere Wissenschaftler wie auch professionelle Praktiker, wobei die Herausgeber mit jeweils mehreren Beiträgen vertreten sind.

Eckhard Jesse dokumentiert in einer vergleichenden Analyse der auf Landesebene erstellten Verfassungsschutzberichte die „Vorverlagerung des Demokratieschutzes“ durch Beobachtung und Aufklärung. Markante Unterschiede in den „Grauzonen“ werden von ihm klar herausgearbeitet. In einer Reihe von praxisbezogenen Empfehlungen kommt ein deutlicher Anwendungsbezug, in wissenschaftssprachlich kaum haltbaren Wendungen wie „CDU-Innenminister“ (S. 26) punktuell eine Orientierung an journalistischer „Schreibe“ zum Ausdruck.

Der gedankenreiche Artikel von Lazaros Miliopoulos zum Anti-Amerikanismus, als ideengeschichtlicher Beitrag etikettiert, ist eher im Umfeld als im Kernbereich des Themenkreises von Extremismus und Demokratie angesiedelt: Vielfältig sind die kulturellen und historischen Aspekte des anregenden Textes. Was jedoch der Leser sich konkret vorstellen soll, wenn der Autor in Islamisten „religiös verkappte Religionsfeinde“ (S. 62) erblickt haben will, bleibt wohl sein Geheimnis: Denn wo soll die Grenze zu originären Anhängern menschenverachtender Religionen verlaufen?

Die ebenfalls ideengeschichtlich angelegte Analyse Evelyn Völkels zu frühen theoretischen Interpretationen des Nationalsozialismus als innerweltliche Religion kommt argumentativ und sprachlich noch etwas unbeholfen daher. Abgesehen von narrativen Längen trägt die Abhandlung auch deutliche Züge der Subjektivität. Eric Voegelin etwa hätte nach Ansicht der Doktorandin besser so argumentieren sollen, wie es ihr plausibel erschienen wäre, meint sie doch, jüngere Zeitabschnitte „scheinen mir rekonstruierbarer“ als die geschichtsmächtigen Spekulationen des Joachim von Fiore, auf die Voegelin argumentativ rekurriert (S. 77 f.). Welche intersubjektiv verbindliche Erkenntnis soll der Leser daraus gewinnen?

Roland Höhne zeichnet die Wandlung der wichtigsten Gruppierungen der italienischen Rechten von der Systemopposition zur Regierungspartei nach - ein Beispiel gelungener Integration, allerdings erst nach einem halben Jahrhundert.

In einem strukturell originellen Teil zum islamischen Fundamentalismus der türkischen Organisation Milli Görüs (IGMG) antworten drei westlich sozialisierte Experten und einer ihrer Funktionsträger auf vier von den Herausgebern gestellte Fragen nach Integrationsgrad und Verfassungsfeindlichkeit der Organisation. Die abschließende Auswertung bleibt allerdings weitgehend eine knappe Zusammenfassung.

Im Abschnitt „Daten, Dokumente, Dossiers“ bietet Eckhard Jesse eine Sekundärauswertung von Wahlstudien in Bezug auf Linkspartei, WASG, REP, DVU und NPD. Uwe Backes dokumentiert organisatorische Entwicklungen und kriminelle Aktionen im Bereich von rechts- und linksextremistischen sowie islamistischen Organisationen. In einem weiteren Eigenbeitrag präsentiert und kommentiert Jesse das 2006 verabschiedete zweite Parteiprogramm der DKP, einer als Vertreterin eines „harten“ Linksextremismus verstandenen, von der „weichen“ Variante der PDS unterschiedenen Partei (S. 212). In seiner Wahlanalyse hatte Jesse hingegen mit Bezug auf SPD und „Die Linke“ von den „beiden demokratischen Linksparteien“" gesprochen (S. 148) - eine bemerkenswert andersartige Klassifizierung, die konzeptionelle Fragen aufwirft. 
Elmar Vieregge analysiert in - besonders für kommunale Entscheidungsträger - aufschlussreicher Weise Grundstücksgeschäfte von Personen aus dem rechtsextremistischen Bereich. Das Auftreten aktionistisch orientierter „Autonomer Nationalisten“, die auf Demonstrationen in "Schwarzen Blocks“ ihre politischen Gegner auf der Linken nachahmen, wird von Christian Menhorn diskutiert. Er sieht hierin eine alters- und regionalspezifische Reaktion auf gesellschaftliche Marginalisierung und polizeiliche Maßnahmen (S. 225).

Das von Karsten Goll vorgelegte Portrait des NPD-Politikers Holger Apfel gilt der persönlichen Dimension von Politik, gerät dabei jedoch unabsichtlich streckenweise bedenklich sympathisch: Im Zuge seiner rechten Sozialisation habe Apfel „eine Reihe junger Leute aus der JN in Niedersachsen“ kennengelernt und „wurde so nach und nach an die Partei herangeführt “ - wie sich das jede Partei für ihre hoffnungsvollen Nachwuchskräfte nur wünschen kann. Der Lehrgangsbeste einer Parteischulung schätzt an ihr rückblickend die „Möglichkeit, in persönlichen Gesprächen Themen zu hinterfragen“, leitende Funktionäre „als Parteifreunde näher kennenzulernen " und Gemeinschaftsbewusstsein mit den anderen Lehrgangsteilnehmern aufzubauen (S. 226 f.). Wenngleich eine Identifizierung mit Apfel vermieden wird und sich am Ende distanziertere Feststellungen finden, klingt das eher nach "gelungener Karriere“ als nach „schiefer Bahn“.

Miroslav Mares Länderstudie zum Extremismus in der Slowakei trägt von ihrem ausgewogenen Ansatz her teilweise Pioniercharakter, bleibt aber nicht nur in ihrem historischen Teil recht deskriptiv. Die jüngst beobachtbare koalitionäre Verbindung linker und rechter Gruppierungen verdient in einem EU-Land indes besondere Aufmerksamkeit.

Sebastian Dietrich zeichnet die unterschiedlichen Positionen von Autoren der mittlerweile eingestellten Zeitschrift „Criticón“ nach, der er eine - problematische - Brückenfunktion zwischen konservativ-demokratischen und rechtsextremen Positionen zuschreibt. Weshalb Dietrich dem langjährigen Herausgeber des Blattes, Caspar Freiherr von Schrenck-Notzing, die Nennung seines korrekten Namens verweigert, bleibt indes ungeachtet aller Vorbehalte gegen die behandelte Publikation unverständlich.

Formal zeigen die Kurzanalysen einen gewissen, nicht ganz überzeugenden Schematismus mit breiten Faktendarlegungen, knapper Gesamtwürdigung und nachklappendem Literaturüberblick. Die längeren, reflektierenden Artikel bieten breite Informationen, wobei allerdings - ebenso wie in den Literaturbesprechungen - die inhaltliche Fokussierung auf „Extremismus und Demokratie“ gelegentlich unscharf wird. Strikte inhaltliche Geschlossenheit wird man von einem Jahrbuch jedoch nicht erwarten können. Der besprochene Band orientiert sich an einer Dokumentation zu Risiken für politische Systeme und einer Darstellung von Trägern extremistischer Weltsicht, deren intellektueller Hintergründe sowie vorhandenen Vernetzungen - ein anspruchsvolles und lohnendes Programm. 